\title{
The yield of tertiary survey in patients admitted for observation after trauma
}

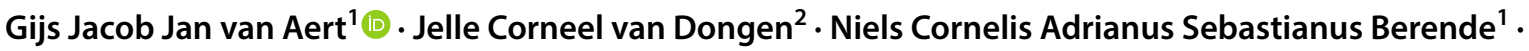 \\ Hendrikus Gerardus Wilhelmus de Groot ${ }^{1}$. Pieter Boele van Hensbroek ${ }^{1}$. Philip Marcel Jozef Schormans ${ }^{1}$. \\ Dagmar Isabella Vos ${ }^{1}$
}

Received: 6 May 2020 / Accepted: 21 August 2020

(c) Springer-Verlag GmbH Germany, part of Springer Nature 2020

\begin{abstract}
Purpose Existing literature on trauma tertiary survey (TTS) focusses on multitrauma patients. This study examines the yield of the TTS in trauma patients with minor (AIS 1) or moderate (AIS 2) injury for which immediate hospitalization is not strictly indicated.

Method A single center retrospective cohort study was performed in a level II trauma center. All hospitalized trauma patients with an abbreviate injury score (AIS) of one or two at the primary and secondary survey were included. The primary outcome was defined as any missed injury found during TTS (Type 1). Secondary outcomes were defined as any missed injury found after TTS but during admission (Type 2); overall missed injury rate; mortality and hospital length of stay.

Results Out of 388 included patients, 12 patients (3.1\%) had a type 1 missed injury. ISS and alcohol consumption were associated with an increased risk for type 1 missed injuries (resp. $O R=1.4, O R=5.49$ ). A type 2 missed injury was only found in one patient. This concerned the only case of trauma related mortality. Approximately one out of five patients were admitted for more than 2 days. These patients were significantly older (66 vs. 41 years, $p<0.001$ ), had a higher ISS ( 4 vs. $3, p=0.007)$ and ASA score, $3-4$ vs. $1-2(42.5 \%$ vs. $12.6 \%, p<0.001)$.

Conclusion TTS showed a low rate of missed injuries in trauma patients with minor or moderate injury. TTS helped to prevent serious damage in two out of 388 patients $(0.5 \%)$. ISS and alcohol consumption were associated with finding missed injury during TTS.
\end{abstract}

Keywords Trauma $\cdot$ Missed injury $\cdot$ Tertiary survey $\cdot$ Patient safety $\cdot$ Quality of care

\section{Introduction}

The Advanced Trauma Life Support (ATLS), as developed by the American College of Surgeons, is a worldwide method for the first treatment of trauma patients. At first, the ATLS approach included two systematic surveys for early diagnosis of both life-threatening and non-life-threatening injuries. During the primary survey, life-threatening conditions are identified and treated in a prioritized sequence $(\mathrm{ABCDE})$. The secondary survey is a more thorough

Gijs Jacob Jan van Aert

gvanaert@amphia.nl

1 Department of Surgery, Amphia Hospital, Molengracht 21, 4818 CK Breda, The Netherlands

2 Department of Surgery, Erasmus MC, Rotterdam, The Netherlands head-to-toe examination with the initiation of definitive care [1]. However, in some trauma patients, these two surveys sometimes fail to identify all sustained injuries. Missed injuries are seen in 1-9\% trauma patients after the primary and secondary survey $[2,3]$. Possible explanations for missing injury at the primary and secondary survey could be the stressfull environment at the emergency department, the complexity of some of the injuries or an altered level of the patients' consciousness.

An additional Trauma Tertiary Survey (TTS), which comprises an in-hospital general physical re-examination and a review of all diagnostic investigations within $24 \mathrm{~h}$, proofed to be successful in reducing the number of missed injuries in multitrauma patients: patients with an Injury Severity Score (ISS) higher than 16 [4-10].

Studies describing the TTS mainly focus on multitrauma patients (ISS $\geq 16$ ). However, trauma patients with an ISS 
scores $<16$ are frequently admitted to the hospital for the treatment of minor injury, social reasons or just for observation after a high energy trauma. Since the tertiary survey is also performed in these cases, even when no injury is found during the primary and secondary survey. The aim of this study was to investigate the outcome of the TTS in this group. Hospitalization raises healthcare costs, the chance of hospital-acquired infections, delirium and other complications [11-13].

We hypothesized that the performance of a TTS in this group might not be beneficial.

\section{Methods}

\section{Study design}

We performed a single center retrospective cohort study at a level 2 trauma center in the Netherlands. The study was approved by the hospital's ethical committee (N2020-0310).

\section{Patients}

All trauma patients, admitted to our hospital between 2015 and 2018, were included when only minor or moderate injury was found during primary and secondary survey. Injury was classified according to the Abbreviated Injury Scale (AIS) Table 1 [14]. Minor injury (AIS =1) was defined as injury for which no operative treatment is needed (e.g., superficial laceration, fracture of the nose, rib contusion). Moderate injury (AIS $=2$ ) was defined as injury for which no immediate treatment was required (e.g., olecranon fracture, patella fracture, distal radius fracture). Reasons for admission could be: no severe injuries found at the primary or secondary survey, but admission just for observation after a high energy trauma; minor injury without requiring immediate in-hospital treatment, but no sufficiënt home care.

Trauma care was provided following ATLS guidelines. All patients were first received at the emergency department's trauma bay, here the primary survey were performed as soon as the patient arrived; a prioritized structured way to identify and treat life-threatening injuries directly (ABCDE). If a patient was stable and the necessary adjuncts had been performed (e.g., X-chest, X-pelvis), the patient was moved to a regular emergency room, where a secondary survey took place (within $1 \mathrm{~h}$ after admission): a thorough head-to-toe examination to identify all injuries. The tertiary survey was performed within $24 \mathrm{~h}$ after admission on the hospital ward. It comprises a general fysical re-examination and review of al investigations, including blood results and imaging, [1].

\section{Data collection}

Demographics collected were: age, gender, BMI, Injury Severity Score (ISS) [15], America Society of Anesthesiologists (ASA)-score [16], mechanism of injury, alcohol consumption (defined as a promillage of $\geq 0.5$ at time of admission at the ER), days of admission, missed injuries and trauma related mortality. All patient data was anonymized to guarantee patient privacy. We dichotomized hospital length of stay with a cut-off point at 2 days to investigate which factors contribute to an unexpected prolonged admission. Although the TTS was performed within $24 \mathrm{~h}$, the cut-off point was set at $48 \mathrm{~h}$ for a more reliable distinction between long hospitalized patients and patients who just exceeded the 24-h window due to-for example-transport problems or post-discharge placement issues.

\section{Outcome measures}

The primary outcome measure in this group was defined as the yield of the tertiary survey, meaning the total number and type of missed injuries found during TTS within $24 \mathrm{~h}$ after admission (Type 1 missed injury [2]). All injuries found after primary and secondary survey and within $24 \mathrm{~h}$ after admission were defined as type 1 missed injury. Secondary outcome measures were defined as missed injuries found after TTS but during the hospital admission (Type 2 missed injury [2]); overall missed injury rate (Type 1 and 2 combined); hospital length of stay; and trauma related mortality. Missed injuries were also classified according to the Abbreviated Injury Scale (AIS) Table 1.

Table 1 Abbreviated injury scale (AIS)

\begin{tabular}{|c|c|}
\hline 1. Minor & Injury for which no treatment is needed (e.g., superficial laceration, fracture of the nose, rib contusion) \\
\hline 2. Moderate & $\begin{array}{l}\text { Injury for which only outpatient treatment was required (e.g., olecranon fracture, distal radius fracture, 1-3 costal fractures without } \\
\text { pneumothorax or hematothorax) }\end{array}$ \\
\hline 3. Serious & $\begin{array}{l}\text { Injury which requires in-hospital Non-ICU treatment. (e.g., open fracture of the humerus, }>3 \text { rib fractures without flail chest, } \\
\text { abdominal organ contusion) }\end{array}$ \\
\hline 4. Severe & Injury which requires ICU observation and/or basic treatment. (e.g., perforated trachea, ruptured spleen, chest-wall perforation) \\
\hline 5. Critical & $\begin{array}{l}\text { Injury which requires intubation, mechanical ventilation or vasopressors for blood pressure support. (spinal cord transection, deep } \\
\text { laceration of kidney of liver) }\end{array}$ \\
\hline A & Not survivable. (e.g., decapitation, torso transection) \\
\hline
\end{tabular}


Patients who underwent surgery during the same admission for any AIS one or two trauma related injury, were excluded for the hospital length of stay analysis, because of the assumption that this direct operative care could result in a longer hospital length of stay. Surgery in this group was defined as surgery performed for injury with an AIS of one or two; meaning that the injury did not require immediate surgery and cosurgery and could have been performed in an elective setting.

\section{Data analysis}

Statistical analysis was performed with SPSS version 25 (IBM Corp., Armonk, NY) for Mac. Non-parametric data was reported as medians noted with interquartile range. Differences between the study groups were tested for statistical significance. Fisher's exact tests were used for categorical data and Mann-Whitney- $U$ tests for continuous variables, as all variables were non-normally distributed. Univariable logistic regression analysis was used to identify possible predictors for a positive TTS.

\section{Results}

Between 2015 and 2018, a total of 4968 trauma patients were admitted to our hospital, 388 patients were included in this study (Fig. 1). Median age was 44 years [IQR 24.00, 64.0] and 136 patients (38.3\%) were female. The median BMI was 25.00 [IQR 22.00, 28.00], 252 patients (64.9\%) had an AIS of 1 and the median ISS was 2.00 [IQR 1.00, 4.00]. Sixty-five patients (18.3\%) had an ASA 3 or 4 score and $25(7.0 \%)$ had consumed alcohol (promillage $\geq 0.5$ ) at the time of admission at the ER. The most frequent mechanism of injury was a high-speed motor vehicle accident, this occurred in 159 (32.8\%) patients (Table 2).

\section{Type 1 missed injury}

Twelve patients (3.1\%) had a type 1 missed injury. Ten injuries were classified as moderate injury (AIS: two) for which conservative treatment was initiated as shown in Table 3. Two of these injuries were classified as severe (AIS: four) and had to be treated operatively. Both of these two patients had an AIS of 2 in two body regions (ISS of eight) at arrival and were admitted for observation because of rib-fractures. One patient showed a decrease of hemoglobin at the TTS, based on a leaking arteria mesenterica. The bleeding was coiled by the intervention radiologist, but a laparotomy was needed to clear the intraperitoneal hematoma. In the other patient, free air was seen during the reassessment of the CTscan. Laparotomy showed a colon perforation for which a resection was performed with a primary anastomosis.

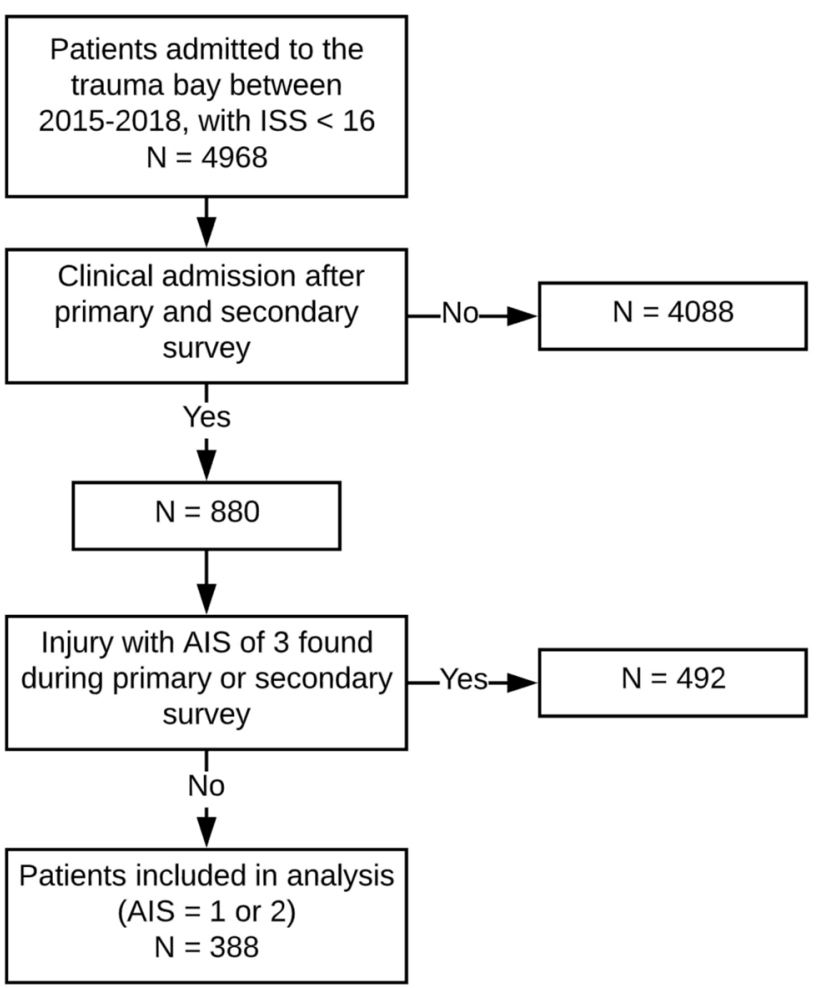

Fig. 1 Inclusion

Table 2 Mechanism of injury (\%)

\begin{tabular}{lr}
\hline MVA high speed & $159(32.8 \%)$ \\
MVA moderate speed & $4(0.8 \%)$ \\
MBA & $21(4.3 \%)$ \\
Fall from height $(>1.5 \mathrm{~m})$ & $39(8.0 \%)$ \\
Fall from standing height & $37(7.6 \%)$ \\
Pedestrian vs. car & $8(1.6 \%)$ \\
Cyclist vs. car & $23(4.7 \%)$ \\
Bike accident & $28(5.8 \%)$ \\
Fall from stairs & $40(8.2 \%)$ \\
Other & $29(6.0 \%)$ \\
\hline
\end{tabular}

MVA motor vehicle accident, High speed: $>30 \mathrm{~km} / \mathrm{h}$, Moderate speed: $<30 \mathrm{~km} / \mathrm{h}$

$M B A$ motor bike accident

Compared to patients without type 1 missed injury, there was no significant difference in gender, BMI, AIS or hospital length of stay. The ISS and the use of alcohol were associated with higher rates of type 1 missed injuries (Table 4).

\section{Type 2 missed injury, overall missed injury rate and mortality}

There was only one case ( $0.3 \%$ ) of a type 2 missed injury; a 65 -year-old male after a high-speed motor vehicle accident 
Table 3 Specifics of type 1 missed injuries found in this study

\begin{tabular}{|c|c|c|c|c|c|}
\hline Patient & Mechanism & $\begin{array}{l}\text { ISS on } \\
\text { arrival }\end{array}$ & Missed Injury & Management & AIS \\
\hline Male 72 years & Fall from height & 8 & Leakage of mesenteric artery & Operative & Severe (4) \\
\hline Female 63 years & MVA high speed & 8 & Perforated colon & Operative & Severe (4) \\
\hline Female 35 years & MVA high speed & 2 & Costal fractures (1-3) & Analgesia & Moderate (2) \\
\hline Female 49 years & MVA high speed & 9 & Costal fractures (1-3) & Analgesia & Moderate (2) \\
\hline Male 69 yeas & MVA high speed & 1 & Impressionfracture L2 & Analgesia, fysiotherapy & Moderate (2) \\
\hline Male 73 years & Fall from standing height & 1 & Costal fractures & Analgesia & Moderate (2) \\
\hline Male 4 years & Pedestrian vs. car & 1 & Clavicula fracture & Immobilization (sling) & Moderate (2) \\
\hline Male 18 years & Bike accident & 5 & Scaphoïd fracture & Immobilization (plaster) & Moderate (2) \\
\hline Male 27 years & Fall from stairs & 5 & Patella fracture & Immobilization (lohmed) & Moderate (2) \\
\hline Female 82 years & Fall from stairs & 1 & Metacarpal fractures & Immobilization (buddy-tape) & Moderate (2) \\
\hline Male 51 years & Other & 6 & Femurfracture (lateral epicondyle) & Immobilisation (plaster) & Moderate (2) \\
\hline
\end{tabular}

MVA Motor vehicle accident, High speed: $>30 \mathrm{~km} / \mathrm{h}$

Table 4 Characteristics of type 1 missed injuries

\begin{tabular}{lllll}
\hline & Negative TTS $N=376$ & Positive TTS N=12 & $p$ & Odds ratio [CI] \\
\hline Age mean (range) & $47.00[26.00,63.00]$ & $50[21.75,71.25]$ & $0.883^{*}$ & $1.001[0.977-1.026]$ \\
Gender = female (\%) & $147(39.1 \%)$ & $4(30.8 \%)$ & $0.772^{* *}$ & $0.779[0.230-2.633]$ \\
BMI [median (IQR)] & $25.00[22.00,28.00]$ & $24.00[21.00,27.00]$ & $0.383^{*}$ & $0.924[0.805-1.061]$ \\
AIS 2 (\%) & $130(34.6 \%)$ & $6(50.0 \%)$ & $0.357^{* *}$ & $1.892[0.598-5.984]$ \\
ISS [median (IQR)] & $3.00[1.00,4.00]$ & $5.00[1.25,7.50]$ & $0.038^{*}$ & $1.389[1.079-1.788]$ \\
Mortality & 0 & 0 & & \\
ASA 3 and 4 & $66(17.6 \%)$ & $3(23.1 \%)$ & $0.454^{* *}$ & $1.566[0.413-5.940]$ \\
Alcohol= yes (\%) & $23(6.1 \%)$ & $3(23.1 \%)$ & $0.039 * *$ & $5.116[1.296-20.194]$ \\
Hospital length of & $1.00[1.00,2.00]$ & $1.50[1.00,6.75]$ & $0.370 *$ & $1.153[1.054-1.262]$ \\
stay*** [median & & & & \\
(IQR)] & & & & \\
\hline
\end{tabular}

TTS trauma tertiary survey, MVA motor vehicle accident, High speed: $>30 \mathrm{~km} / \mathrm{h}$, moderate speed: $<30 \mathrm{~km} / \mathrm{h}$

*Mann-Whitney- $U$-test

**Fisher's exact
(MVA). During the primary and secondary survey only a minor back injury was found (AIS of one). Tertiary survey showed no missed injury. The patient remained hospitalized because of general weakness without evidence of neurological or surgical pathology. Three days after admission he developed neurological symptoms (ataxia of the upper limbs en sensory loss of the lower limbs) and an MRI of the spine was made. It showed myelumcompression caused by anteroposition of cervical vertebrae six on seven (not detectable at the CT-scan performed at the ER). The patient was immediately transferred to a level 1 neurosurgical specialized hospital, were decompressive surgery of the cervical cord was performed in combination with a spondylodesis of cervical vertebrae 6 and 7. Despite this operative management the patient developed a tetraplegia and became dependant on mechanical ventilation. One month later the treatment was stopped because of the patient's wishes. This was the only case of trauma related mortality in this study. The overall missed injury rate in this study, type 1 and 2 combined, was $3.4 \%$.

\section{Hospital length of stay ( $\leq 2$ days vs. $>2$ days)}

Out of the 388 patients, 20 underwent surgery (Table 5) during the primary admission and were therefore excluded for the hospital length of stay analysis. Of the remaining 366 patients, 73 patients $(19.9 \%)$ were hospitalized for more than 2 days (Table 6). These patients were significantly older compared to patients who were admitted for 1 or 2 days (66 vs. 41 years old, $p>0.001$ ), had a higher AIS (AIS $=2: 49.3 \%$ vs. $29.4 \%, p=0.002$ ) and ISS ( 4.00 vs. 3.00 , $p=0.007)$ and also the ASA-score was significantly higher (ASA 3 and 4: $42.5 \%$ vs. $12.6 \%, p>0.001$ ). All other variables did not differ significantly. 
Table 5 Surgery in included patients

\begin{tabular}{lr}
\hline Wound care/debridement & 7 \\
Fixation of radial fracture & 4 \\
Fixation of humeral fracture & 2 \\
Fixation of tibial fracture & 2 \\
Fixation of patella fracture & 1 \\
Fixation of clavicle fracture & 1 \\
Fixation of mandibular & 1 \\
K-wire fixation of metacarpal five fracture & 2 \\
Laparotomy & 2 \\
Total & 22 \\
\hline
\end{tabular}

\section{Discussion}

Tertiary Trauma Survey proofed to be succesfull in multitrauma patients, but less is known of its effectiveness in patients with minor trauma. The question arises whether the tertiary survey is usefull in this group and which factors contribute to the chance for missed injury. To our knowledge this is the first study reporting on the outcome of the tertiary survey in a trauma population without severe injury (AIS of $\geq 3$ ) at the primary and secondary ATLS survey.

We observed $3.1 \%$ type 1 missed injury, this can be considered as low. In a systematic review conducted by Keijzer et al. [2] $4.3 \%$ type 1 missed injuries were found. However, they note that there was great heterogenity between the studies included in their review. Type 1 missed injury rates, varied from $1.5 \%$ to $19.3 \%$ (with an outlier of even 65\%). Moreover, the two largest studies in this review $[18,19]$ (who included more than 9000 subjects, reporting a missed inury rate of approximately $1.5 \%)$, only recorded missed injury when this resulted in a change of treatment or required intervention. This leads to an underestimation of the type 1 missed injury rates.

In the findings of our study there appears to be an association between type 1 missed injury and the ISS score. Previous studies [2, 17, 20-22] have shown an association in patients with an ISS $\geq 16$ (polytrauma). But now, even in patients with an ISS $<16$, the higher the score, the higher the chance of a missed injury. We expect this to be due to a more trustworthy physical examination when there is less distracting injury.

Alcohol consumption was also associated with higher chances of finding missed injuries at the tertiary survey. Aaland and Smith [23] have noted this link before. In their study $39 \%$ out of 56 trauma patients with missed injury were intoxicated ( 0.63 positive predictive index). Alcohol consumption leads to an altered reaction during physical examination. Therefore injuries can be missed more easily.

Two out of twelve type 1 missed injuries (17\%) were finally classified as severe (AIS 4) and even needed operative intervention. This is comparible to previous research of Giannakopoulos et al. [24] and Vles et al. [17] in which operative management was required in respectively $20 \%$ and $25 \%$ of the patients with missed injuries. Concerning the severity of the injuries found, conducting the TTS helped to prevent more serious damage in these two patients.

Type 2 missed injury was found only in one patient $(0.3 \%)$. Though the injury was severe, it appeared that it was not detectable on routine imaging and could not have been detected earlier by tertiary survey due to a late onset of symptoms. Only one study, Biffl et al. [25], reported on type 2 missed injury in particular, they found a $1.5 \%$ type 2 missed injury rate. However, this rate was found in
Table 6 Characteristics of hospital length of stay ( 1 or 2 days vs. more than 2 days)

\begin{tabular}{lllll}
\hline & $\begin{array}{l}\text { Hospital stay<2 days } \\
\mathrm{N}=293\end{array}$ & $\begin{array}{l}\text { Hospital stay }>2 \text { days } \\
\mathrm{N}=73\end{array}$ & $P$ & Odds ratio [CI] \\
\hline Age [median (IQR)] & $41.00[24.00,58.00]$ & $66.00[52.50,80.00]$ & $>0.001^{*}$ & $1.052[1.037-1.067]$ \\
Gender = female (\%) & $104(35.5 \%)$ & $36(49.3 \%)$ & $0.032^{* *}$ & $1.768[1.054-2.967]$ \\
BMI (median [IQR]) & $25.00[22.00,28.00]$ & $26.00[22.00,29.00]$ & $0.472^{*}$ & $1.018[0.959-1.082]$ \\
AIS 2 (\%) & $86(29.4 \%)$ & $36(49.3 \%)$ & $0.002^{*}$ & $2.342[1.388-3.952]$ \\
ISS [median (IQR)] & $3.00[1.00,4.00]$ & $4.00[2.00,5.00]$ & $0.007^{*}$ & $1.207[1.066-1.366]$ \\
Mortality & 0 & 0 & & \\
ASA 3 and 4 & $37(12.6 \%)$ & $31(42.5 \%)$ & $>0.001^{* *}$ & $5.107[2.865-9.104]$ \\
Alcohol = yes (\%) & $24(8.2 \%)$ & $2(2.7 \%)$ & $0.129^{* *}$ & $0.316[0.073-1.368]$ \\
Clinical significant & $7(2.4 \%)$ & $3(4.1 \%)$ & $0.424^{* *}$ & $1.751[0.442-6.943]$ \\
injury found during & & & & \\
TTS (\%) & & & & \\
\hline
\end{tabular}

TTS trauma tertiary survey, MVA motor vehicle accident, High speed: $>30 \mathrm{~km} / \mathrm{h}$, Moderate speed: $<30 \mathrm{~km} / \mathrm{h}$

*Mann-Whitney- $U$-test

**Fisher's exact 
trauma patients with ISS scores higher than 16, who were admitted to the intensive care unit (ICU). The explanation for this very low percentage type 2 missed injury is the accurately performed primary, secondary and tertiary survey, who together detecte most missed injuries.

The overall missed injury rate (type 1 and 2 combined) in this study was $3.4 \%$. This seems to be low compared to existing literature. A review conducted by Pfeifer et al. [19] showed missed injury rates varying from $1.3 \%$ to $39 \%$, Keijzer et al. [2] found an overall missed injury rate of 5.8\% and Giannakopoulos et al. [24] reported 8.2\%. Importantly, these studies investigated all trauma patients including polytrauma patients (ISS $>16$ ).

Twenty-one percent of our patients were admitted for more than 2 days. These patients were on average older and had a higher ISS and ASA-score. This is in line with the study conducted by Kashkooe et al. [26], a cross-sectional study with a population of approximately 14,000 patients. They indicated that patients of higher age were more susceptible to have a longer hospital length of stay. Ramarkably, one out of five patients is admitted to the hospital for more than 2 days, while there is no serious injury at admission and few missed injuries are found during TTS. An explanation for this might be that pre-existant comorbidities and higher chances of in-hospital complications such as pneumonia, result in a longer hospital stay. In addition, we expect that the absence of adequate home care in older patients is an important factor. Improving home care in frail elderly could result in earlier discharge and might prevent in-hospital complications.

Although the percentage of severe injury found at the TTS is low [2 out of 388 patients $(0.5 \%)$ ], it can be concluded that performing a TTS in this study's population still is usefull in some cases. But considering the overall findings of this study, we recommend to maintain a high threshold in the admission of trauma patients for TTS when the following criteria are met: Patients without AIS of 3 or more found at primary or secondary survey, ISS lower than eight, ASA 1 or 2, no alcohol intoxication, the patient has the wish to go home and adequate social support can be provided. Discharging a patient instead of hospitalisation can contribute to the well-being of the patient and to the saving of medical expenses.

The results of this study should be interpreted within the context of the study design; a retrospective singlecenter cohort study. However, this study is conducted in a large level 2 trauma center were patients with all different socio-economic and ethnic backgrounds are seen. This makes our study population representative to other countries. Future research should focus on predicting variables of a positive tertiary survey in these trauma patients and a standardized guideline should be developed to supports physicians in deciding whether to admit a patient or sending them home safely.

\section{Conclusion}

Trauma tertiary survey showed low percentages of missed injury (3.1\%) in trauma patients without serious injury (AIS of 3 or higher) upon admission. Tertiary trauma survey revealed severe missed injuries in 2 cases (0.5\%). ISS and alcohol consumption were associated with higher chances of finding missed injuries during tertiary survey and a higher age and a ASA-score (3 or 4) often results in an unexpected longer admission. The use of the tertiary survey in trauma patients with an ASA score of 1 or 2, a low ISS upon arrival, without an alcohol intoxication and with adequate home care can be discussed.

Funding Not applicable.

Data availability Yes.

\section{Compliance with ethical standards}

Conflict of interest The authors declare that they have no conflicts of interest.

\section{References}

1. Brasel KJ. Advanced trauma life support (ATLS®): the ninth edition. J Trauma Acute Care Surg. 2013;74(5):1363-6. https://doi. org/10.1097/TA.0b013e31828b82f5.

2. Keijzers GB, Giannakopoulos GF, Del Mar C, Bakker FC, Geeraedts LMG. The effect of tertiary surveys on missed injuries in trauma: a systematic review. Scand J Trauma Resusc Emerg Med. 2012;20:1-9. https://doi.org/10.1186/1757-7241-20-77.

3. Thomson CB, Greaves I. Missed injury and the tertiary trauma survey. Injury. 2008;39(1):107-14. https://doi.org/10.1016/j.injur y.2007.07.030.

4. Enderson BL, Reath DB, Meadors J, Dallas W, Deboo JM, Maull KI. The tertiary trauma survey: a prospective study of missed injury. J Trauma Inj Infect Crit Care. 1990;30(6):666-70. https:// doi.org/10.1097/00005373-199006000-00002.

5. Zamboni C, Yonamine AM, Faria CEN, Filho MAM, Christian RW, Mercadante MT. Tertiary survey in trauma patients: avoiding neglected injuries. Injury. 2014;45(S5):S14-S1717. https://doi. org/10.1016/S0020-1383(14)70014-2.

6. Hajibandeh S, Hajibandeh S, Idehen N. Meta-analysis of the effect of tertiary survey on missed injury rate in trauma patients. Injury. 2015;46(12):2474-82. https://doi.org/10.1016/j.injur y.2015.09.019.

7. Brooks A, Holroyd B, Riley B. Missed injury in major trauma patients. Injury. 2004;35(4):407-10. https://doi.org/10.1016/ S0020-1383(03)00219-5.

8. Hoff WS, et al. Formalized radiology rounds: the final component of the tertiary survey. J Trauma Inj Infect Crit Care. 2004;56(2):291-5. https://doi.org/10.1097/01.TA.0000105924 .37441 .31 
9. Janjua KJ, Sugrue M, Deane SA. Prospective evaluation of early missed injuries and the role of tertiary trauma survey. J Trauma Inj Infect Crit Care. 1998;44(6):1000-7. https://doi. org/10.1097/00005373-199806000-00012.

10. Houshian S, Larsen MS, Holm C. Missed injuries in a level I trauma center. J Trauma. 2002;52(4):715-9. https://doi. org/10.1097/00005373-200204000-00018.

11. Schimmel EM. the Hazards of Hospitalization. Ann Intern Med. 1964;60:100-10. https://doi.org/10.7326/0003-4819-60-1-100.

12. Hwabejire JO, et al. Excessively long hospital stays after trauma are not related to the severity of illness: let's aim to the right target! JAMA Surg. 2013;148(10):956-61. https://doi.org/10.1001/ jamasurg.2013.2148.

13. Mathew PJ, et al. The burden of excess length of stay in trauma patients. Am J Surg. 2018;216(5):881-5. https://doi.org/10.1016/j. amjsurg.2018.07.044.

14. Gennarelli T. Abbreviated injury scale 2005: update 2008. Barrington Ill.: Association for the Advancement of Automative Medicine; 2008.

15. "The injury severity score: a method for describing patients with multiple injuries and evaluating emergency care-PubMed." [Online]. https://pubmed.ncbi.nlm.nih.gov/4814394/. (Accessed 12-Jul-2020).

16. "ASA Physical Status Classification System I American Society of Anesthesiologists (ASA)." [Online]. https://www.asahq.org/ standards-and-guidelines/asa-physical-status-classification-syste m. (Accessed 12-Jul-2020).

17. Vles WJ, Veen EJ, Roukema JA, Meeuwis JD, Leenen LPH. Consequences of delayed diagnoses in trauma patients: a prospective study. J Am Coll Surg. 2003;197(4):596-602. https://doi. org/10.1016/S1072-7515(03)00601-X.
18. Huynh TT, Blackburn AH, McMiddleton-Nyatui D, Moran KR, Thomason MH, Jacobs DG. An initiative by midlevel providers to conduct tertiary surveys at a level I trauma center. J Trauma Inj Infect Crit Care. 2010;68(5):1052-5. https://doi.org/10.1097/ TA.0b013e3181d87789.

19. Pfeifer R, Pape H-C. Missed injuries in trauma patients: a literature review. Patient Saf Surg. 2008;2(1):1-6. https://doi. org/10.1186/1754-9493-2-20.

20. Ferree S, Houwert RM, Van Laarhoven JJEM, Smeeing DPJ, Leenen LPH, Hietbrink F. Tertiary survey in polytrauma patients should be an ongoing process. Injury. 2016;47(4):792-6. https:// doi.org/10.1016/j.injury.2015.11.040.

21. Stevens NM. "in the Tertiary Survey," vol. 6; 12: pp. 1-6, 2018.

22. Buduhan G, McRitchie DI. Missed injuries in patients with multiple trauma. J Trauma. 2000;49(4):600-5. https://doi. org/10.1097/00005373-200010000-00005.

23. Aaland MO, Smith K. Delayed diagnosis in a rural trauma center. Surgery. 1996;120(4):774-8. https://doi.org/10.1016/s0039 -6060(96)80030-4 (discussion 778-9).

24. Giannakopoulos GF, et al. Missed injuries during the initial assessment in a cohort of 1124 level-1 trauma patients. Injury. 2012;43(9):1517-21. https://doi.org/10.1016/j.injury.2011.07.012.

25. Biffl WL, Harrington DT, Cioffi WG. Implementation of a tertiary trauma survey decreases missed injuries. J Trauma. 2003;54(1):38-44. https://doi.org/10.1097/00005373-20030100000005 .

26. Kashkooe A, Yadollahi M, Pazhuheian F. What factors affect length of hospital stay among trauma patients? A single-center study, southwestern Iran. Chinese J Traumatol. 2020. https://doi. org/10.1016/j.cjtee.2020.01.002. 